\title{
THE SIMPLIFIED WEIGHTED SUM FUNCTION AND ITS AVERAGE SENSITIVITY
}

\author{
JIYOU LI AND CHU LUO
}

\begin{abstract}
In this paper we simplify the definition of the weighted sum Boolean function which used to be inconvenient to compute and use. We show that the new function has essentially the same properties as the previous one. In particular, the bound on the average sensitivity of the weighted sum Boolean function remains unchanged after the simplification.
\end{abstract}

\section{INTRODUCTION}

In previous study, the weighted sum function has a simple but not very clean structure. With a residue ring modulo a prime, the explicit definition of this function can be given using the weighted sum as follows $\left[21\right.$. Let $m \in \mathbb{Z}^{+}=\{1,2,3, \ldots\}$ and prime number $p \geq m$ where no other prime numbers are between $p$ and $m$. For vector $X=\left(x_{1}, x_{2}, \ldots, x_{m}\right) \in \mathbb{Z}_{2}^{m}$, where $\mathbb{Z}_{2}=\{0,1\}$, let $u(X)$ be the least positive integer which satisfies

$$
u(X)=\sum_{k=1}^{m} k x_{k}(\bmod p), 1 \leq u(X) \leq p .
$$

Then the weighted sum function $g(X)$ is defined as

$$
g(X)=\left\{\begin{array}{l}
x_{u(X)}, \quad 1 \leq u(X) \leq m \\
x_{1}, \quad \text { otherwise }
\end{array}\right.
$$

This function was used to study read-once branching programs by P. Savický and S. Žák 21]. It was also used to demonstrate the exponential improvement from conventional read-once branching programs to quantum ones by M. Sauerhoff in [18, see also [19].

To simplify the definition of the previous weighted sum function, we define a new function $f(X)$ as follows. For $X=\left(x_{0}, x_{1}, \ldots, x_{m-1}\right) \in \mathbb{Z}_{2}^{m}$, denote

$$
s(X)=\sum_{k=0}^{m-1} k x_{k}(\bmod m),
$$

and define the new weighted sum function

$$
f(X)=x_{s(X)} .
$$

It is worth noting that this new function $f(X)$ is more convenient to compute and use than $g(X)$. One particular reason for the prime modulus in the previous function $g(X)$ is that there are nice results and structures in prime fields. In this

This work is supported by the National Science Foundation of China (11001170) and the National Science Foundation of Shanghai Municipal (13ZR1422500).

1 
paper we call such $f(X)$ the simplified weighted sum function. Note that when $m$ is prime then the two definitions are the same.

We believe that in many cases this new simplified function $f(X)$ has similar properties as the previous one $g(X)$. We give two examples to support this claim. As the first example, $g(X)$ was used in 21] to establish a lower bound of read-once branching programs. We show that a similar lower bound holds for $f(X)$.

A Boolean function is called $k$-mixed if for any two distinct partial assignments of any given $k$ variables, the two subfunctions on the remaining $m-k$ variables are distinct. This notion was first introduced by Jukna [13. For more applications on more functions, see [2, 3, 11, 20, 26].

One of the key ingredients in 21] is the following theorem, first proved by Simon and Szegedy [25].

Theorem 1.1. If $h(X)$ is a k-mixed Boolean function, then every 1-branching program for $h(X)$ has size at least $2^{k}-1$.

The authors then showed that the weighted sum function $g(X)$ of $m$ variables is $(m-o(m))$-mixed, by applying the following result in additive combinatorics and established the desired lower bound.

Theorem 1.2 (Dias da Silva and Hamidoune, 9]). Let $\epsilon>0$ be a fixed constant. Then, for every large enough $p$ and $A \subseteq \mathbb{Z}_{p}$ with $|A| \geq(2+\epsilon) \sqrt{p}$, and for every $b \in \mathbb{Z}_{p}$, there is a subset $B \subseteq A$ such that the sum of the elements of $B$ is equal to $b$.

The work of Freeze, Gao and Geroldinger in 2009 generalized this result to the general cyclic group case.

Theorem 1.3 (Freeze, Gao and Geroldinger, [10]). Let $d$ be the smallest prime divisor of $m$. Then, for every $A \subseteq \mathbb{Z}_{m}$ with $|A| \geq \frac{m}{d}+d-2$, and for every $b \in \mathbb{Z}_{m}$, there is a subset $B \subseteq A$ such that the sum of the elements of $B$ is equal to $b$.

Similarly one can use this result to prove that the simplified weighted sum function $f(X)$ is $\left(m\left(1-\frac{1}{d}\right)-d\right)$-mixed and thus establish the similar lower bound for $f(X)$ as well. This bound is pretty good especially when $m$ 's smallest prime divisor $d$ is large compared to $m$. For instance, if we take $m$ to be a product of two primes which are close enough, then the bound is as good as the prime case.

Interestingly, this argument can be applied to some other topics. As the second example, the $k$-mixed property was used to give a lower bound for Boolean circuits. For details we refer to 3 .

We then determine the average sensitivity of this newly defined function $f(X)$ and show that it also satisfies the Shparlinski's conjecture [24] which says that the average sensitivity of $f(X)$ is asymptotically $m / 2$. We introduce the main concepts of this conjecture in the following.

For an input $X=\left(x_{0}, x_{1}, \ldots, x_{m-1}\right)$, the sensitivity $\sigma_{s, X}(f)$ on $X$ denotes the number of variables such that flipping one of these variables will shift the value of f. Explicitly,

$$
\sigma_{s, X}(f)=\sum_{i=0}^{m-1}\left|f(X)-f\left(X^{(i)}\right)\right|
$$

where $X^{(i)}=\left(x_{0}, \ldots, x_{i-1}, 1-x_{i}, x_{i+1} \ldots, x_{m-1}\right)$ is the vector assignment after flipping the $i$-th coordinate in $X$. The sensitivity $\sigma_{s}(f)$ of $f(X)$ denotes the maximum of $\sigma_{s, X}(f)$ on vector $X$ in $\mathbb{Z}_{2}^{m}$ and the average sensitivity $\sigma_{a v}(f)$ is the mean 
value of sensitivity on every possible input, i.e.,

$$
\sigma_{a v}(f)=2^{-m} \sum_{X \in \mathbb{Z}_{2}^{m}} \sum_{i=0}^{m-1}\left|f(X)-f\left(X^{(i)}\right)\right| .
$$

Sensitivity, together with a more general concept called block sensitivity, is a useful measure to predict the complexity of Boolean functions. It has recently drawn extensive attention, for instance [1, 4, 5, 6, 7, 8, 14, 17, 22, 23, 24. For a good survey on the main unsolved problems on sensitivity, please refer to 12 .

In 24 Shparlinski addressed the average sensitivity problem of the previous weighted sum function $g(X)$ and obtained a lower bound from a nontrivial bound on its Fourier coefficients using exponential sums methods. He also developed several conjectures on the average sensitivity of the weighted sum function and the bounds of the Fourier coefficients. Explicitly, one conjecture was that the average sensitivity of $g(X)$ on $m$ variables is not less than $\left(\frac{1}{2}+o(1)\right) m$. In the same paper he gave a proof that the average sensitivity is greater than $\gamma m$, where constant $\gamma$ satisfies $\gamma \approx 0.0575$.

By applying a new sieving technique, in 14 the first author gave an asymptotic counting formulas of the subset sums over prime fields and thus confirmed the Shparlinski's conjecture on the average sensitivity of the weighted sum function.

In this paper we extend this result for the simplified weighted sum function $f(X)$. That is, for $f(X)$ with $m$ variables, the average sensitivity of $f(X)$ is exactly $(1 / 2+o(1)) m$.

In addition, we also compute the weight of $f(X)$. We prove that the weight of $f(X)$ on $m$ variables is exactly $2^{m-1}(1+o(1))$. Thus, $f(X)$ is an asymptotically balanced function.

This paper is organized as follows. In Section 2 we present a sieve formula. By applying this formula, we give a series of formulas for counting subsets sums over cyclic groups in Section 3. The proof of the main results is given in Section 4. We also list several further questions in Section 5 .

Notation. For $x \in \mathbb{R}$, let $(x)_{0}=1$ and $(x)_{k}=x(x-1) \cdots(x-k+1)$ for $k \in \mathbb{Z}^{+}=\{1,2,3, \ldots\}$. For $k \in \mathbb{N}=\{0,1,2, \ldots\}$ define the binomial coefficient $\left(\begin{array}{l}x \\ k\end{array}\right)=\frac{(x)_{k}}{k !}$.

\section{A Distinct COORDinate SIEVING FORMUla}

For the purpose of our proof, we briefly introduce a sieving formula discovered by Li-Wan [15], which significantly improves the classical inclusion-exclusion sieving. We cite it here without any proof. For details and related applications, we refer to [15, 16.

Let $S_{k}$ be the symmetric group on $k$ elements. It is well known that every permutation $\tau \in S_{k}$ factorizes uniquely as a product of disjoint cycles and each fixed point is viewed as a trivial cycle of length 1 . For $\tau \in S_{k}$, define $\operatorname{sign}(\tau)=(-1)^{k-l(\tau)}$, where $l(\tau)$ is the number of cycles of $\tau$ including the trivial cycles.

Theorem 2.1. Suppose $X$ is a finite set of vectors of length $k$ over an alphabet set D. Define $\bar{X}=\left\{\left(x_{1}, x_{2}, \ldots, x_{k}\right) \in X \mid x_{i} \neq x_{j}, \forall i \neq j\right\}$. Let $f\left(x_{1}, x_{2}, \ldots, x_{k}\right)$ be a complex valued function defined over $X$ and $F=\sum_{x \in \bar{X}} f\left(x_{1}, x_{2}, \ldots, x_{k}\right)$. Then

$$
F=\sum_{\tau \in S_{k}} \operatorname{sign}(\tau) F_{\tau}
$$


where

$$
X_{\tau}=\left\{\left(x_{1}, \ldots, x_{k}\right) \in X, x_{i_{1}}=\cdots=x_{i_{a_{1}}}, \cdots, x_{l_{1}}=\cdots=x_{l_{a_{s}}}\right\},
$$

for a permutation $\tau=\left(i_{1} i_{2} \cdots i_{a_{1}}\right)\left(j_{1} j_{2} \cdots j_{a_{2}}\right) \cdots\left(l_{1} l_{2} \cdots l_{a_{s}}\right)$ with $1 \leq a_{i}, 1 \leq i \leq s$ and $F_{\tau}=\sum_{x \in X_{\tau}} f\left(x_{1}, x_{2}, \ldots, x_{k}\right)$.

Note that the symmetric group $S_{k}$ acts on $D^{k}$ naturally by permuting coordinates. That is, for $\tau \in S_{k}$ and $x=\left(x_{1}, x_{2}, \ldots, x_{k}\right) \in D^{k}, \tau \circ x=\left(x_{\tau(1)}, x_{\tau(2)}, \ldots, x_{\tau(k)}\right)$. A subset $X$ in $D^{k}$ is said to be symmetric if for any $x \in X$ and any $\tau \in S_{k}, \tau \circ x \in X$. In particular, if $X$ is symmetric and $f$ is a symmetric function under the action of $S_{k}$, we then have the following formula which is simpler than (2.1).

Corollary 2.2. Let $C_{k}$ be the set of conjugacy classes of $S_{k}$. If $X$ is symmetric and $f$ is symmetric, then

$$
F=\sum_{\tau \in C_{k}} \operatorname{sign}(\tau) C(\tau) F_{\tau},
$$

where $C(\tau)$ is the number of permutations conjugate to $\tau$.

For the purpose of evaluating the above summation, we need several combinatorial formulas. A permutation $\tau \in S_{k}$ is said to be of type $\left(c_{1}, c_{2}, \cdots, c_{k}\right)$ if $\tau$ has exactly $c_{i}$ cycles of length $i$ and that $\sum_{i=1}^{k} i c_{i}=k$. Let $N\left(c_{1}, c_{2}, \ldots, c_{k}\right)$ be the number of permutations in $S_{k}$ of type $\left(c_{1}, c_{2}, \ldots, c_{k}\right)$ and it is well-known that

$$
N\left(c_{1}, c_{2}, \ldots, c_{k}\right)=\frac{k !}{1^{c_{1}} c_{1} ! 2^{c_{2}} c_{2} ! \cdots k^{c_{k}} c_{k} !} .
$$

Lemma 2.3. Define the generating function

$$
C_{k}\left(t_{1}, t_{2}, \ldots, t_{k}\right)=\sum_{\sum i c_{i}=k} N\left(c_{1}, c_{2}, \ldots, c_{k}\right) t_{1}^{c_{1}} t_{2}^{c_{2}} \cdots t_{k}^{c_{k}} .
$$

If $t_{1}=t_{2}=\cdots=t_{k}=q$, then we have

$$
\begin{aligned}
C_{k}(q, q, \ldots, q) & =\sum_{\sum i c_{i}=k} N\left(c_{1}, c_{2}, \ldots, c_{k}\right) q^{c_{1}} q^{c_{2}} \cdots q^{c_{k}} \\
& =(q+k-1)_{k} .
\end{aligned}
$$

In another case, if $t_{i}=q>0$ for $d \mid i$ and $t_{i}=s>0$ for $d \nmid i$, then we have

$$
C_{k}(\overbrace{s, \cdots, s}^{d-1}, q, \overbrace{s, \cdots, s}^{d-1}, q, \cdots) \leq(s+(q-s) / d+k-1)_{k} .
$$

Proof. By the definition of the generating function,

$$
\begin{aligned}
C_{k}(\overbrace{s, \cdots, s}^{d-1}, q, \overbrace{s, \cdots, s}^{d-1}, q, \cdots) & =\sum_{\sum i c_{i}=k} N\left(c_{1}, c_{2}, \cdots, c_{k}\right) s^{c_{1}} s^{c_{2}} \cdots q^{c_{d}} s^{c_{d+1}} \cdots \\
& =\left[\frac{u^{k}}{k !}\right] e^{u s+u^{2} \cdot \frac{s}{2}+\cdots+u^{d-1} \cdot \frac{s}{d-1}+u^{d} \cdot \frac{q}{d}+u^{d+1} \cdot \frac{s}{d+1} \cdots} \\
& =\left[\frac{u^{k}}{k !}\right] e^{-s \log (1-u)-\frac{q-s}{d} \log \left(1-u^{d}\right)} \\
& =\left[\frac{u^{k}}{k !}\right] \frac{1}{(1-u)^{s}\left(1-u^{d}\right)^{\frac{q-s}{d}}} .
\end{aligned}
$$




$$
\begin{aligned}
& \leq\left[\frac{u^{k}}{k !}\right] \frac{1}{(1-u)^{s}(1-u)^{\frac{q-s}{d}}} \\
& =(s+(q-s) / d+k-1)_{k} .
\end{aligned}
$$

\section{Subset Sum Problem in a Subset of the Cyclic Groups}

Let $\mathbb{Z}_{m}$ be the cyclic group of $m$ elements. Let $D \subseteq \mathbb{Z}_{m}$ be a nonempty subset of cardinality $n$. Let $\widehat{\mathbb{Z}}_{m}$ be the group of additive characters of $\mathbb{Z}_{m}$, i.e, all the homomorphisms from $\mathbb{Z}_{m}$ to the nonzero complex numbers $\mathbb{C}^{*}$. Note that $\widehat{\mathbb{Z}}_{m}$ is isomorphic to $\mathbb{Z}_{m}$. Let $\chi_{0}$ be the trivial character. For an additive character $\chi$ in $\widehat{\mathbb{Z}}_{m}$, define $s_{\chi}(D)=\sum_{a \in D} \chi(a)$ and $\Phi(D)=\max _{\chi \in \widehat{\mathbb{Z}}_{m}, \chi \neq \chi_{0}}\left|s_{\chi}(D)\right|$. Let $N(k, b, D)$ be the number of $k$-subsets $T \subseteq D$ such that $\sum_{x \in T} x=b$. In the following theorem we will give an asymptotic bound for $N(k, b, D)$ which ensures $N(k, b, D) \sim \frac{1}{m}\left(\begin{array}{l}n \\ k\end{array}\right)$ when $\mathbb{Z}_{m}-D$ is not too large compared with $\mathbb{Z}_{m}$.

Theorem 3.1. Let $N(k, b, D)$ be defined as above.

$$
\left|N(k, b, D)-m^{-1}\left(\begin{array}{l}
n \\
k
\end{array}\right)\right| \leq \frac{1}{m} \sum_{\substack{1<r \leq m \\
r \mid m}} \phi(r)\left(\begin{array}{c}
\frac{n+\Phi(D)}{r}+k-1 \\
k
\end{array}\right),
$$

where $d$ is the smallest prime divisor of $m$ and $\phi(r)$ is the Euler function.

Proof. We notice that the proof is essentially similar to the proof of main theorem in 14, and is a bit more involved since $n$ may not be a prime number. Let $X=D \times D \times \cdots \times D$ be the Cartesian product of $k$ copies of $D$. Let $\left.\bar{X}=\left\{\left(x_{1}, x_{2}, \ldots, x_{k}\right) \in D^{k} \mid x_{i} \neq x_{j}, \forall i \neq j\right\}\right\}$. It is clear that $|X|=n^{k}$ and $|\bar{X}|=(n)_{k}$. Applying the orthogonal relation $\sum_{\psi \in \widehat{\mathbb{Z}}_{m}} \psi(a)=0$ for $a \not \equiv 0(\bmod m)$ and $\sum_{\psi \in \widehat{\mathbb{Z}}_{m}} \psi(a)=m$ for $a \equiv 0(\bmod m)$, we have

$$
\begin{aligned}
k ! N(k, b, D) & =m^{-1} \sum_{\left(x_{1}, x_{2}, \ldots x_{k}\right) \in \bar{X}} \sum_{\chi \in \widehat{\mathbb{Z}}_{m}} \chi\left(x_{1}+x_{2}+\cdots+x_{k}-b\right) \\
& =m^{-1}(n)_{k}+m^{-1} \sum_{\chi \neq \chi_{0}} \sum_{\left(x_{1}, x_{2}, \cdots x_{k}\right) \in \bar{X}} \chi\left(x_{1}\right) \chi\left(x_{2}\right) \cdots \chi\left(x_{k}\right) \chi^{-1}(b) \\
& =m^{-1}(n)_{k}+m^{-1} \sum_{\chi \neq \chi_{0}} \chi^{-1}(b) \sum_{\left(x_{1}, x_{2}, \ldots x_{k}\right) \in \bar{X}} \prod_{i=1}^{k} \chi\left(x_{i}\right) .
\end{aligned}
$$

Denote $f_{\chi}(x)=f_{\chi}\left(x_{1}, x_{2}, \ldots, x_{k}\right)=\prod_{i=1}^{k} \chi\left(x_{i}\right)$. For $\tau \in S_{k}$, let

$$
F_{\tau}(\chi)=\sum_{x \in X_{\tau}} f_{\chi}(x)=\sum_{x \in X_{\tau}} \prod_{i=1}^{k} \chi\left(x_{i}\right),
$$

where $X_{\tau}$ is defined as in (2.2). Obviously $X$ is symmetric and $f_{\chi}\left(x_{1}, x_{2}, \ldots, x_{k}\right)$ is normal on $X$. Applying (2.3) in Corollary 2.2, we get

$$
k ! N(k, b, D)=m^{-1}(n)_{k}+m^{-1} \sum_{\chi \neq \chi_{0}} \chi^{-1}(b) \sum_{\tau \in C_{k}} \operatorname{sign}(\tau) C(\tau) F_{\tau}(\chi),
$$


where $C_{k}$ is the set of conjugacy classes of $S_{k}, C(\tau)$ is the number of permutations conjugate to $\tau$. If $\tau$ is of type $\left(c_{1}, c_{2}, \ldots, c_{k}\right)$, then

$$
\begin{aligned}
F_{\tau}(\chi) & =\sum_{x \in X_{\tau}} \prod_{i=1}^{k} \chi\left(x_{i}\right) \\
& =\sum_{x \in X_{\tau}} \prod_{i=1}^{c_{1}} \chi\left(x_{i}\right) \prod_{i=1}^{c_{2}} \chi^{2}\left(x_{c_{1}+2 i}\right) \cdots \prod_{i=1}^{c_{k}} \chi^{k}\left(x_{c_{1}+c_{2}+\cdots+k i}\right) \\
& =\prod_{i=1}^{k}\left(\sum_{a \in D} \chi^{i}(a)\right)^{c_{i}} \\
& =n^{\sum c_{i} m_{i}(\chi)} s_{\chi}(D)^{\sum c_{i}\left(1-m_{i}(\chi)\right)},
\end{aligned}
$$

where $m_{i}(\chi)=1$ if $\chi^{i}=1$ and otherwise $m_{i}(\chi)=0$.

Now suppose order $(\chi)=r$ with $d \leq r \mid m$. Note that $C(\tau)=N\left(c_{1}, c_{2}, \ldots, c_{k}\right)$ and by Lemma 2.3 we have

$$
\begin{aligned}
& \sum_{\tau \in C_{k}} \operatorname{sign}(\tau) C(\tau) F_{\tau}(\chi) \\
& \leq \sum_{\tau \in C_{k}} C(\tau) n^{\sum c_{i} m_{i}(\chi)} \Phi(D)^{\sum c_{i}\left(1-m_{i}(\chi)\right)} \\
& \leq k !\left(\begin{array}{c}
\frac{n+\Phi(D)}{r}+k-1 \\
k
\end{array}\right) .
\end{aligned}
$$

Similarly, if $\operatorname{order}(\chi)$ is greater than $k$, then

$$
\sum_{\tau \in C_{k}} \operatorname{sign}(\tau) C(\tau) F_{\tau}(\chi) \leq k !\left(\begin{array}{c}
\Phi(D)+k-1 \\
k
\end{array}\right) .
$$

Note that there are $\phi(r)$ characters of order $r$. Summing over all nontrivial characters, we obtain

$$
\left|N(k, b, D)-m^{-1}\left(\begin{array}{l}
n \\
k
\end{array}\right)\right| \leq \frac{1}{m} \sum_{\substack{1<r \leq m \\
r \mid m}} \phi(r)\left(\begin{array}{c}
\frac{n+\Phi(D)}{r}+k-1 \\
k
\end{array}\right),
$$

where $\phi(r)$ is the Euler function. This completes the proof.

Corollary 3.2. We have

$$
\left|N(k, b, D)-m^{-1}\left(\begin{array}{l}
n \\
k
\end{array}\right)\right| \leq\left(\begin{array}{c}
\frac{n+\Phi(D)}{d}+k-1 \\
k
\end{array}\right),
$$

where $d$ is the minimum prime divisor of $m$.

Corollary 3.3. If $|D|=m-c$ and $c$ is a positive constant, noting that $\Phi(D) \leq c$ we have

$$
\left|N(k, b, D)-m^{-1}\left(\begin{array}{c}
m-c \\
k
\end{array}\right)\right| \leq\left(\begin{array}{c}
\frac{m}{d}+k-1 \\
k
\end{array}\right),
$$

where $d$ is the minimum prime divisor of $m$.

A simple combinatorial argument on sums of binomial coefficients gives 
Corollary 3.4. Let $n=m-o(m)$. Let $N(b, D)=\sum_{k=0}^{n} N(k, b, D)$ be the number of subsets in $D$ which sums to $b$. Then $N(b, D)=\frac{2^{n}}{m}(1+o(1))$.

\section{Average Sensitivity}

In [14, the weight and the average sensitivity of $g(X)$ are computed. We now generalize these results to the simplified function $f(X)$. We first compute the weight of $f(X)$.

Theorem 4.1. Let $f(X)$ be defined as above. Then we have

$$
w t(f)=2^{m-1}(1+o(1)) \text {. }
$$

In other words, $f(X)$ is an asymptotically balanced function.

Proof. By applying Corollary 3.4 we have

$$
\begin{aligned}
w t(f(X))=\sum_{X \in \mathbb{Z}_{2}^{m}} f(X) & =\sum_{s=0}^{m-1} \sum_{X \in \mathbb{Z}_{2}^{m}, s(X)=s, x_{s}=1} 1 \\
& =\sum_{s=0}^{m-1} N\left(0, \mathbb{Z}_{m} \backslash\{s\}\right) \\
& =\sum_{s=0}^{m-1} \frac{1}{m} 2^{m-1}(1+o(1)) \\
& =2^{m-1}(1+o(1))
\end{aligned}
$$

In 24] Shparlinski studied $\sigma_{a v}(g(X))$ and raised the following question:

Question 4.2. Is it true that for the function given by 1.1 we have

$$
\sigma_{a v}(g(X)) \geq\left(\frac{1}{2}+o(1)\right) m ?
$$

In the same paper Shparlinski gave a lower bound by obtaining a nontrivial bound on the Fourier coefficients of $g(X)$ via analytical methods. He proved in the same paper that this value is greater than $\gamma m$, where $\gamma \approx 0.0575$ is a constant. Li answered this question in [14.

Theorem 4.3. Let $\sigma_{a v}(g)$ be the average sensitivity of the previous weighted sum function $g(X)$. Then

$$
\sigma_{a v}(g(X))=\left(\frac{1}{2}+o(1)\right) m .
$$

Here we prove that this conjecture still holds for $f(X)$ :

Theorem 4.4. Let $\sigma_{a v}(f)$ be the average sensitivity of the simplified weighted sum function $f(X)$. Then

$$
\sigma_{a v}(f(X))=\left(\frac{1}{2}+o(1)\right) m .
$$

Proof. Since we have the symmetry between the bits 1 and 0 , for simplicity we just need to consider the number of bit changes from 0 to 1 . Thus by Corollary 3.4

$$
2^{m-1} \sigma_{a v}(f(X))=\sum_{X \in \mathbb{Z}_{2}^{m}} \sum_{i=0}^{m-1}\left|f(X)-f\left(X^{(i)}\right)\right|
$$




$$
\begin{aligned}
= & \sum_{s=0}^{m-1} \sum_{X \in \mathbb{Z}_{2}^{m}, s(X)=s, x_{s}=1} \sum_{i=0}^{m-1}\left|1-f\left(X^{(i)}\right)\right| \\
& +\sum_{s=0}^{m-1} \sum_{X \in \mathbb{Z}_{2}^{n}, s(X)=s, x_{s}=0} \sum_{i=0}^{m-1}\left|0-f\left(X^{(i)}\right)\right| \\
= & \sum_{i=0}^{m-1} \sum_{s=0}^{m-1} \sum_{X \in \mathbb{Z}_{2}^{m}, s(X)=s, x_{i}=0, x_{s}=1, x_{s+i}=0} 1 \\
& +\sum_{i=0}^{m-1} \sum_{s=0}^{m-1} \sum_{X \in \mathbb{Z}_{2}^{m}, s(X)=s, x_{i}=0, x_{s}=1, x_{s+i}=1} 1 \\
= & \sum_{i=0}^{m-1} \sum_{s=0}^{m-1} N\left(0, \mathbb{Z}_{m} \backslash\{i, s+i, s\}\right)+\sum_{i=0}^{m-1} \sum_{s=0}^{m-1} N\left(0, \mathbb{Z}_{m} \backslash\{i, s-i, s\}\right) \\
= & \sum_{i=0}^{m-1} \sum_{s=0}^{m-1} \frac{2^{m-2}}{m}(1+o(1)) \\
= & m 2^{m-2}(1+o(1)) .
\end{aligned}
$$

Finally we have

$$
\sigma_{a v}(f(X))=\left(\frac{1}{2}+o(1)\right) m
$$

\section{Further QUeStions}

In 24] Shparlinski studied the Fourier coefficients of the weighted sum function.

Definition 5.1. Let $h(X)$ be a Boolean function from $\{0,1\}^{m}$ to $\{0,1\}$. The Fourier coefficient of $h(X)$ at a is defined by

$$
\widehat{h}(a)=\frac{1}{2^{m}} \sum_{X \in\{0,1\}^{m}}(-1)^{h(X)+a \cdot X} .
$$

Shparlinski raised the following conjecture which is stronger than his conjecture on the average sensitivity.

Conjecture 5.2 (Shparlinski, 24]). For the previous weighted sum function $g(X)$, we have

$$
\max |\widehat{g}(a)|=2^{\left(-\frac{1}{2}+o(1)\right) m} .
$$

Shparlinski proved in 24] that

$$
\max |\widehat{g}(a)| \leq 2^{(-\rho+o(1)) m},
$$

where

$$
\rho=\frac{4}{\pi \ln 2} \mathcal{L}\left(\frac{\pi}{4}\right), \quad \mathcal{L}(x)=-\int_{0}^{x} \ln \cos \theta d \theta .
$$

Note that $\rho \approx 0.1587$. Currently this is still the best result.

We compute the value of $\max |\widehat{f}(a)|$ over $0<m<22$ using a computer program. The results are shown in Table 1. Note again that when $m$ is prime then $f(X)=$ $g(X)$. The experimental results indicate that Shparlinski's conjecture may not be true. It will be amazing to obtain the true bounds on both $\max |\widehat{g}(a)|$ and $\max |\widehat{f}(a)|$. 
TABLE 1. The relationship between the variable number $m$ and the maximal Fourier coefficients of $f(X)$ over $0<m<22$. Decimals are rounded to three decimal places.

\begin{tabular}{|c|c|c|}
\hline$m$ & $\max |\widehat{f}(a)|$ & $m^{-1} \log _{2} \max |\widehat{f}(a)|$ \\
\hline 1 & 1 & 0 \\
\hline 2 & 0.5 & -0.5 \\
\hline 3 & 0.5 & -0.333 \\
\hline 4 & 0.75 & -0.104 \\
\hline 5 & 0.75 & -0.083 \\
\hline 6 & 0.438 & -0.199 \\
\hline 7 & 0.469 & -0.156 \\
\hline 8 & 0.281 & -0.229 \\
\hline 9 & 0.305 & -0.191 \\
\hline 10 & 0.227 & -0.214 \\
\hline 11 & 0.146 & -0.252 \\
\hline 12 & 0.209 & -0.188 \\
\hline 13 & 0.093 & -0.264 \\
\hline 14 & 0.086 & -0.253 \\
\hline 15 & 0.159 & -0.177 \\
\hline 16 & 0.067 & -0.244 \\
\hline 17 & 0.059 & -0.240 \\
\hline 18 & 0.119 & -0.171 \\
\hline 19 & 0.053 & -0.224 \\
\hline 20 & 0.050 & -0.216 \\
\hline 21 & 0.089 & -0.166 \\
\hline
\end{tabular}

Instead of the Shparlinski's conjecture, we propose a new conjecture:

Conjecture 5.3. For the newly defined weighted sum function $f(X)$, we have

$$
\max |\widehat{f}(a)|=2^{(-\rho+o(1)) m} .
$$

Acknowledgements. The authors wish to thank the referees for their careful reading of the paper and many useful comments which helped us to improve this paper. The first author also wishes to thank the hospitality of the Department of Mathematics, MIT, while revising this paper.

\section{REFERENCES}

[1] K. Amano, Tight bounds on the average sensitivity of $k-C N F$, Theory Comput. 7 (2011), $45-48$.

[2] A. Andreev, J. Baskakov, A. Clementi and J. Rolim, Small pseudo-random sets yield hard functions: new tight explicit lower bounds for branching programs, in: Proc. 26th ICALP (1999), 179-189.

[3] K. Amano and J. Tarui, A well-mixed function with circuit complexity $5 n$ : tightness of the Lachish-Raz-type bounds, Theoret. Comput. Sci. 412 (2011), 1646-1651.

[4] A. Bernasconi, Sensitivity vs. block sensitivity (an average-case study), Inform. Process. Lett. 59 (1996), 151-157.

[5] A. Bernasconi, C. Damm and I. Shparlinski, The average sensitivity of square-freeness, Comput. Complexity 9 (2000), 39-51. 
[6] R. B. Boppana, The average sensitivity of bounded-depth circuits, Inform. Process. Lett. 63 (1997), 257-261.

[7] H. Buhrman and R. de Wolf, Complexity measures and decision tree complexity: a survey, Complexity and logic (Vienna, 1998), Theoret. Comput. Sci. 288 (2002), 21-43.

[8] D. Canright, S. Gangopadhyay, S. Maitra and P. Stanica, Laced Boolean functions and subset sum problems in finite fields, Discrete Applied Mathematics, 159 (2011), 1059-1069.

[9] J.A. Dias da Silva and Y.O. Hamidoune, Cyclic spaces for Grassmann derivatives and additive theory, Bull. London Math. Soc. 26 (1994),140-146.

[10] M. Freeze, W.D. Gao and A. Geroldinger, The critical number of finite abelian groups. J. Number Theory 129 (2009), 2766-2777.

[11] A. Gál, A simple function that requires exponential size read-once branching programs, Inform. Process. Lett. 62 (1997), 13-16.

[12] P. Hatami, R. Kulkarni and D. Pankratov, Variations on the Sensitivity Conjecture, Theory of Computing Library Graduate Surveys, 4 (2011), 1-27.

[13] S. Jukna, Entropy of contact circuits and lower bounds on their complexity, Theoret. Comput. Sci. 57 (1988), 113-129.

[14] J. Li, On the average sensitivity of the weighted sum function, Inform. Process. Lett. 112 (2012), 143-148.

[15] J. Li and D. Wan, A new sieve for distinct coordinate counting, Science in China Series A, 53 (2010), 2351-2362.

[16] J. Li and D. Wan, Counting subsets of finite Ablelian groups, J. Combin. Theory Ser. A, 19 (2012),170-182.

[17] D. Rubinstein, Sensitivity vs. block sensitivity of Boolean functions, Combinatorica 15 (1995), 297-299.

[18] M. Sauerhoff, Randomness versus nondeterminism for read-once and read-k branching programs, STACS 2003, 307-318, Lecture Notes in Comput. Sci., 2607, Springer, Berlin, 2003.

[19] M. Sauerhoff and D. Sieling, Quantum branching programs and space-bounded nonuniform quantum complexity, Theoret. Comput. Sci., 334 (2005), 177-225.

[20] P. Savicky and S. Zak, A large lower bound for 1-branching programs, in: ECCC TR96-036 Rev.01, 1996.

[21] P. Savicky and S. Zak, A read-once lower bound and a $(1,+k)$-hierarchy for branching programs, Theoret. Comput. Sci. 238 (2000), 347-362.

[22] D. Scheder and L.Y. Tan, On the average sensitivity and density of $k$-CNF formulas, Approximation, randomization, and combinatorial optimization, 683-698, Lecture Notes in Comput. Sci., 8096, Springer, Heidelberg, 2013.

[23] Y. Shi, Lower bounds of quantum black-box complexity and degree of approximating polynomials by influence of Boolean variables, Inform. Process. Lett. 75 (2000), 79-83.

[24] Igor. E. Shparlinski, Bounds on the Fourier coefficients of the weighted sum function, Inform. Process. Lett. 103 (2007), 83-87.

[25] J. Simon and M. Szegedy, A new lower bound theorem for read-only-once branching programs and its applications, Advances in computational complexity theory, 183C193, DIMACS Ser. Discrete Math. Theoret. Comput. Sci., 13, Amer. Math. Soc., Providence, RI, 1993.

[26] I. Wegener, Branching programs and binary decision diagrams, SIAM Monographs on Discrete Mathematics and Applications, SIAM, 2000.

Department of Mathematics, Shanghai Jiao Tong University, Shanghai, P.R. China

E-mail address: lijiyou@sjtu.edu.cn

Department of Computer Science and Engineering, University of Oulu, Oulu, FinLAND

E-mail address: chu.luo@ee.oulu.fi 